\title{
Gray per Hour
}

National Cancer Institute

\section{Source}

National Cancer Institute. Gray per Hour. NCI Thesaurus. Code C158295.

A unit of absorbed radiation dose rate defined as the number of Grays per hour. 